\title{
ON PROJECTION-DIFFERENCE ANALOGUES OF THE IDENTITY OPERATOR
}

\author{
N. A. STRELKOV
}

pr. Dzerzhinskogo, 3, kv. 173, 150033 Yaroslavl', Russia

In this note an answer is given to the question of A. B. Borisov, formulated during the Second Soviet School "Contemporary Problems of Numerical Analysis" (Khar'kov, October 1990). The question concerns the structure of projectivegrid analogues of the operator $\partial / \partial t-\Delta$; in a slightly reformulated form the problem leads to constructing the approximating subspaces with the optimal rate of approximation in the metric of $W_{2}^{1}$ such that the projective-grid analogue of the identity operator has the same five point stencil as the simplest projective-grid analogue of the Laplace operator.

Below the negative answer to this question is given, i.e., it is shown that there exists no finite element subspace of the space $W_{2}^{1}$ whose approximation properties are optimal with respect to the rate and which generates an analogue of the unit operator with the five point "cross-type" stencil. Moreover, a full description of the projective-grid analogues of the identity operator is given.

Let $\mathbb{E}_{2}$ be the two-dimensional Euclidean space, and $\mathbb{Z}_{2}$ the set of integervalued vectors in $\mathbb{E}_{2}$. We consider the prolongation operators of the grid functions of the form

$$
R \bar{u}(x)=\sum_{\alpha \in \mathbb{Z}_{2}} u_{\alpha}^{h} \psi_{\alpha}^{h}(x),
$$

where $\bar{u}$ is a grid function defined on the square grid $h \mathbb{Z}_{2}$ with step $h, u_{\alpha}^{h}$ is the value of $\bar{u}$ at the node $\alpha h, \alpha \in \mathbb{Z}_{2}$, and the functions $\psi_{\alpha}^{h}$ are generated by a fixed function $\psi \in W_{2}^{1}\left(\mathbb{E}_{2}\right)$ supported on $[-1,1] \times[-1,1]$ :

$$
\psi_{\alpha}^{h}(x)=\psi(x / h-\alpha), \quad \alpha \in \mathbb{Z}_{2}, x \in \mathbb{E}_{2} .
$$

1991 Mathematics Subject Classification: 65N06, 35J20.

This work was supported in part by the International Science Foundation.

The paper is in final form and no version of it will be published elsewhere. 
In addition, only those functions $\psi$ will be considered for which the prolongation operators of the form (1) generated by them have the following property: For an arbitrary $u \in W_{2}^{2}\left(\mathbb{E}_{2}\right)$ we can find a grid function $\bar{u}$ such that

$$
\begin{gathered}
\left\{h^{2} \sum_{\alpha \in \mathbb{Z}_{2}}\left(u_{\alpha}^{h}\right)^{2}\right\}^{1 / 2} \leq K\|u\|_{L_{2}\left(\mathbb{E}_{2}\right)}, \\
\|u-R \bar{u}\|_{W_{2}^{s}\left(\mathbb{E}_{2}\right)} \leq C_{s} h^{2-s}\|u\|_{W_{2}^{2}\left(\mathbb{E}_{2}\right)}, \quad 0 \leq s \leq 1,
\end{gathered}
$$

where $K$ and $C_{s}$ do not depend on $u$ and $h$.

The projective-grid analogue $E$ of the unit operator is defined by the identity

$$
(R \bar{u}, R \bar{v})_{L_{2}\left(\mathbb{E}_{2}\right)}=h^{2} \sum_{\alpha \in \mathbb{Z}_{2}}(E u)_{\alpha}^{h} v_{\alpha}^{h}
$$

for all grid functions $\bar{u}$ and $\bar{v}$.

As is shown in [3] (cf. also [4], Theorem 1.1), a function $\psi \in W_{2}^{1}\left(\mathbb{E}_{2}\right)$ whose support is $[-1,1] \times[-1,1]$ generates a prolongation operator $(1)$ with the properties (2), (3) if and only if the Fourier transform $\widehat{\psi}$ of $\psi$ has the following properties:

(A) $\widehat{\psi}$ is an entire function of exponential type 1 (see, e.g., [2])

(B) $\widehat{\psi}(x)\left(1+|x|^{2}\right)^{1 / 2} \in L_{2}\left(\mathbb{E}_{2}\right)$,

(C) $\widehat{\psi}(0) \neq 0, \widehat{\psi}(2 \pi \alpha)=\mathcal{D}_{1} \widehat{\psi}(2 \pi \alpha)=\mathcal{D}_{2} \widehat{\psi}(2 \pi \alpha)=0$ for each $\alpha \in \mathbb{Z}_{2}, \alpha \neq 0$.

Since multiplication of a function $\psi$ by a nonzero constant does not change the properties of an operator $R$ of the form (1), later on we assume that in the property $(\mathrm{C})$ the normalization condition $\widehat{\psi}(0)=1$ is satisfied.

For convenience we introduce the notation

$$
g(t)=\frac{2 \sin \frac{t}{2}}{t}, \quad t \in \mathbb{E}_{1},
$$

and we describe in a constructive way all the functions satisfying conditions (A) $-(\mathrm{C})$.

Using the results of the paper [5] and carrying out an argument which differs from the proof of Lemma 5.2 in [4] only in nonsignificant details, we can easily prove that every function satisfying conditions $(\mathrm{A})-(\mathrm{C})$ must have the form

$$
\widehat{\psi}(z)=g\left(z_{1}\right) g\left(z_{2}\right)\left\{g\left(z_{1}\right) g\left(z_{2}\right)+\sum_{p \in \mathbb{Z}_{2}^{0}} c_{p} g\left(z_{1}-2 \pi p_{1}\right) g\left(z_{2}-2 \pi p_{2}\right)\right\}
$$

where $\mathbb{Z}_{2}^{0}=\left\{p \in \mathbb{Z}_{2}: p_{1} p_{2} \neq 0\right\}$, and $c_{p}=4 \pi^{2}(-1)^{p_{1}+p_{2}} p_{1} p_{2} \mathcal{D}_{1} \mathcal{D}_{2} \widehat{\psi}(2 \pi p)$.

Therefore, a function $\psi$ generates a prolongation operator (1) with properties (2), (3) if and only if its Fourier transform $\widehat{\psi}$ is of the form (5).

Let us now answer the question which analogues of the unit operator can be generated by such functions. For this purpose, we apply Theorem 1.1 from [4] to the case under consideration. According to this theorem, the operator $E$ defined 
by identity (4) is completely described by the behaviour of the function

$$
G(x)=\sum_{\alpha \in \mathbb{Z}_{2}}|\widehat{\psi}(x+2 \pi \alpha)|^{2} .
$$

Using the equalities

$$
\begin{gathered}
\sum_{k=-\infty}^{\infty}\left(\frac{\sin x}{x+\pi k}\right)^{4}=1-\frac{2}{3} \sin ^{2} x, \\
\sum_{k=-\infty}^{\infty} \frac{\sin ^{2} x}{(x+\pi k)^{3}(x-\pi p+\pi k)}=-\frac{1}{\pi p}\left(\frac{1}{\pi p}+\cot x\right), \\
\sum_{k=-\infty}^{\infty} \frac{\sin ^{2} x}{(x+\pi k)^{2}(x-\pi p+\pi k)(x-\pi q+\pi k)}=\frac{1}{\pi^{2} p q}\left(1+\delta_{p}^{q}\right),
\end{gathered}
$$

where $p \neq 0, q \neq 0$ (see, e.g., [1]), we deduce that if $\widehat{\psi}$ is of the form (5) then

$$
\begin{aligned}
G(x)= & \left(1-\frac{2}{3} \sin ^{2} \frac{x_{1}}{2}\right)\left(1-\frac{2}{3} \sin ^{2} \frac{x_{2}}{2}\right) \\
& +\sin ^{2} \frac{x_{1}}{2} \sin ^{2} \frac{x_{2}}{2}\left\{\sum_{p \in \mathbb{Z}_{2}^{0}} \frac{c_{p}+\bar{c}_{p}}{\pi^{2} p_{1} p_{2}}\left(\frac{1}{\pi p_{1}}+\cot \frac{x_{1}}{2}\right)\left(\frac{1}{\pi p_{2}}+\cot \frac{x_{2}}{2}\right)\right. \\
& \left.+\frac{1}{\pi^{4}} \sum_{q, p \in \mathbb{Z}_{2}^{0}} \frac{c_{p} \bar{c}_{q}}{p_{1} q_{1} p_{2} q_{2}}\left(1+\delta_{p_{1}}^{q_{1}}\right)\left(1+\delta_{p_{2}}^{q_{2}}\right)\right\} .
\end{aligned}
$$

Applying now equality (1.9) and Remark 1.8 from the paper [4], which sets the connection between the function $G$ and the difference operators, we obtain

$$
\begin{aligned}
(E u)_{\alpha}^{h}= & u_{\alpha}^{h}+\left\{\frac{h^{2}}{6}\left(\bar{\partial}_{1} \partial_{1}+\bar{\partial}_{2} \partial_{2}\right)+i A h^{3} \widetilde{\partial}_{1} \bar{\partial}_{2} \partial_{2}\right. \\
& \left.+i B h^{3} \bar{\partial}_{1} \partial_{1} \widetilde{\partial}_{2}+C h^{2} \widetilde{\partial}_{1} \widetilde{\partial}_{2}+D h^{4} \bar{\partial}_{1} \partial_{1} \bar{\partial}_{2} \partial_{2}\right\} u_{\alpha}^{h},
\end{aligned}
$$

where $\partial_{k} u(x)=h^{-1}\left[u\left(x+h e_{k}\right)-u(x)\right], \bar{\partial}_{k} u(x)=h^{-1}\left[u(x)-u\left(x-h e_{k}\right)\right]$ are the first forward and backward differences in the direction of $e_{k}, \widetilde{\partial}_{k}=\frac{1}{2}\left(\partial_{k}+\bar{\partial}_{k}\right)$, and the real numbers $A, B, C, D$, depending on the sequence $\left\{c_{p}\right\}_{p \in \mathbb{Z}_{2}^{0}}$ from formula (5), have the form

$$
\begin{aligned}
& A=A(c)=\frac{1}{4 \pi^{3}} \sum_{p \in \mathbb{Z}_{2}^{0}} \frac{a_{p}}{p_{1} p_{2}^{2}}, \quad B=B(c)=\frac{1}{4 \pi^{3}} \sum_{p \in \mathbb{Z}_{2}^{0}} \frac{a_{p}}{p_{1}^{2} p_{2}}, \\
& C=C(c)=-\frac{1}{2 \pi^{2}} \sum_{p \in \mathbb{Z}_{2}^{0}} \frac{a_{p}}{p_{1} p_{2}}, \\
& D=D(c)=\frac{1}{36}+\frac{1}{8 \pi^{4}} \sum_{p \in \mathbb{Z}_{2}^{0}} \frac{a_{p}}{p_{1}^{2} p_{2}^{2}}+\frac{1}{16 \pi^{4}}\left\{\left|\sum_{p \in \mathbb{Z}_{2}^{0}} \frac{c_{p}}{p_{1} p_{2}}\right|^{2}\right.
\end{aligned}
$$




$$
\left.+\sum_{p_{1} \neq 0} \frac{1}{p_{1}^{2}}\left|\sum_{p_{2} \neq 0} \frac{c_{p}}{p_{2}}\right|^{2}+\sum_{p_{2} \neq 0} \frac{1}{p_{2}^{2}}\left|\sum_{p_{1} \neq 0} \frac{c_{p}}{p_{1}}\right|^{2}+\sum_{p \in \mathbb{Z}_{2}^{0}} \frac{\left|c_{p}\right|^{2}}{p_{1}^{2} p_{2}^{2}}\right\}
$$

(here $a_{p}=\operatorname{Re} c_{p}, b_{p}=\operatorname{Im} c_{p}$ ).

Let us remark that if we additionally require that the function $\psi$ defined on $\mathbb{E}_{2}$, whose Fourier transform has the form (5), admits only real values, then $c_{-p}=\bar{c}_{p}$ for all $p \in \mathbb{Z}_{2}^{0}$; in this case $A(c)=B(c)=0$ and equality (6) can be simplified.

It follows from (6) that at an arbitrary knot $x \in \mathbb{Z}_{2}$ the following equality is satisfied:

$$
E u(x)=\sum_{k_{1}=-1}^{1} \sum_{k_{2}=-1}^{1} A_{k_{1}, k_{2}} u\left(x_{1}+k_{1} h, x_{2}+k_{2} h\right),
$$

where $A_{0,0}=\frac{1}{3}+4 D, A_{ \pm 1,0}=\frac{1}{6}-2 D \mp i A, A_{0, \pm 1}=\frac{1}{6}-2 D \mp i B, A_{1, \pm 1}=$ $D \pm \frac{1}{4} C+\frac{i}{2}(A \pm B), A_{-1, \pm 1}=D \mp \frac{1}{4} C-\frac{i}{2}(A \mp B)$.

Hence, the set of projective-grid analogues of the unit operator which are generated by the prolongation operators of the form (1) with conditions (2), (3) has the form (7).

With the application of the equality

$$
\sum_{p \in \mathbb{Z}_{2}^{0}} \frac{1}{p_{1}^{2} p_{2}^{2}}=\frac{\pi^{4}}{9}
$$

$D(c)$ can be rewritten in the form

$$
\begin{aligned}
D(c)= & \frac{1}{48}+\frac{1}{16 \pi^{4}}\left\{\left|\sum_{p \in \mathbb{Z}_{2}^{0}} \frac{c_{p}}{p_{1} p_{2}}\right|^{2}+\sum_{p_{1} \neq 0} \frac{1}{p_{1}^{2}}\left|\sum_{p_{2} \neq 0} \frac{c_{p}}{p_{2}}\right|^{2}\right. \\
& \left.+\sum_{p_{2} \neq 0} \frac{1}{p_{2}^{2}}\left|\sum_{p_{1} \neq 0} \frac{c_{p}}{p_{1}}\right|^{2}+\sum_{p \in \mathbb{Z}_{2}^{0}} \frac{\left(a_{p}+1\right)^{2}+b_{p}^{2}}{p_{1}^{2} p_{2}^{2}}\right\},
\end{aligned}
$$

which implies that the set of the values of $D(c)$ as a function of the sequence $c=\left\{c_{p}\right\}_{p \in \mathbb{Z}_{2}^{0}}$ is the half-line $\left[\frac{1}{48}, \infty\right)$. Therefore $D(c) \neq 0$ and for any choice of $c=\left\{c_{p}\right\}_{p \in \mathbb{Z}_{2}^{0}}$ at least two of the numbers $A_{1,1}, A_{1,-1}, A_{-1,1}, A_{-1,-1}$ are different from zero. This means that there exists no projection-difference analogue of the unit operator which has the five point cross-type stencil. It is clear that, by choosing the sequence $c=\left\{c_{p}\right\}_{p \in \mathbb{Z}_{2}^{0}}$, we can always achieve that $D(c)=\frac{1}{12}$, $A(c)=B(c)=0$, from which it follows that $A_{ \pm 1,0}=A_{0, \pm 1}=0$, i.e., the operator $E$ has the five point stencil of the type "skew cross" (moreover, in this case we can also obtain the three point stencil $\left\{x,\left(x_{1}+h, x_{2}+h\right),\left(x_{1}-h, x_{2}-h\right)\right\}$ if $C(c)=\frac{1}{3}$ or the stencil $\left\{x,\left(x_{1}+h, x_{2}-h\right),\left(x_{1}-h, x_{2}+h\right)\right\}$ if $\left.C(c)=-\frac{1}{3}\right)$. However, as follows from the results of the paper [6], each of the projective-grid analogues of the Laplace operator has either the five point cross-type stencil or completely filled 9-point stencil; hence in every case the union of the stencils of the unit operator and the Laplace operator consists at least of seven points. Analogous 
conclusions can also be obtained in the case when we assume in advance that the function $\psi$ is real-valued.

Clearly, the existence of a cross-type stencil can be achieved by weakening the conditions for the approximation properties of the prolongation operators (or by extending the support of the function $\psi$ ); for this question see the final considerations of the paper [6].

\section{References}

[1] I. S. Gradshteı̆n and I. M. Ryzhik, Tables of Integrals, Sums, Series and Products, Nauka, Moscow 1971 (in Russian).

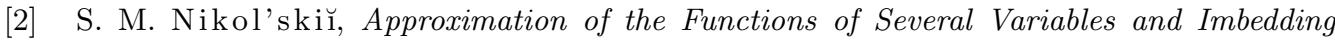
Theorems, Nauka, Moscow 1977 (in Russian).

[3] G. Strang and G. Fix, A Fourier analysis of the finite element method, in: Proc. CIME Summer School, Italy 1971.

[4] N. A. Strelkov, On the relationship between difference and projection-difference methods, in: Numerical Analysis and Mathematical Modeling, Banach Center Publ. 24, PWN, Warszawa 1990, 355-377.

[5] - Spline-trigonometric bases in $L_{2}$ and interpolation of entire functions of exponential type, Mat. Zametki 32 (6) (1982), 835-840 (in Russian).

[6] -, On projection-difference analogues of the Laplace operator, Zh. Vychisl. Mat. i Mat. Fiz. 21 (5) (1981), 1326-1328 (in Russian). 